in the superstructure. Childish defects really represent lines of direction from the life of the psyche, and are signals indicating peripheral or central inferiority which has not been overcome. We can always find attentiveness of the central superstructure proportionately distributed on the part of the normal organ, disproportionately distributed and increased according to the organic over-compensation in the inferior organ, more easily aroused but less productive by reason of unsuccessful compensation, insufficient or absent in cases of lasting central inferiority.

This work refers all phenomena of neuroses and psycho-neuroses back to organ inferiority, to the degree and nature of the not quite successful central compensation, and to the compensatory disturbances which enter into the matter.

IV. J. A. ERSKINE.

\title{
2. Neurology.
}

Pupillary and Reflex Disturbances in 275 Cases of Neurosyphilis. (Journ. of Nerv. and Ment. Dis., August, 1920.) Lowrey, L. G., and Benedicl, Mary $K$.

In this study of all cases of neuro-syphilis seen during a year and a half, only those cases were utilised where the presence of neuro-syphilis had been proved. The clinical diagnoses were: general paresis, I 86 cases ; tabo-paresis, I 2 ; tabes, 8 ; juvenile paresis, 4 ; neuro-syphilis (not further specified), 65. The authors did not find that the ArgyllRobertson pupil occurred in every case of tabes and tabo-paresis; 43 per cent. only of paresis, tabo-paresis, juvenile paresis and tabes showed the phenomenon. Of the whole series $40^{\circ} 7$ per cent. reacted in this way. Impaired pupillary reaction was found in $7 \mathrm{r}^{\circ} 3$ per cent. of cases $-40^{\circ} 7$ per cent. Argyll-Robertson; 1 I 7 per cent. spastic and 8.3 per cent. sluggish both to light and on acconmodation; 10.5 per cent. with impaired reaction not further specified-leaving 28.7 per cent. of cases in which the reactions were normal. Inequality and irregularity of pupils were common. Irregular pupils are of more diagnostic import than unequal pupils, since the number of possible causes is less. These abnormal reactions do not inevitably mean neuro-syphilis, as the ArgyllRobertson pupil temporarily occurs in alcoholics, the completely spastic pupil in arterio-sclerosis cerebri, unequal and irregular pupils in iritis. Too much reliance, therefore, must not be placed on the pupils in neuro-syphilis, especially in view of the 29 per cent. of normal pupils in cases of paresis.

In 70 per cent. of the cases some type of abnormal tendon reflex response was found. All cases of tabes and tabo-paresis and 70 per cent. of the paretic cases showed some reflex disorder. The knee-jerk showed an alteration in 43 per cent. of all cases, and in $4 \mathrm{I} \cdot 7$ per cent. of paresis. A lost reflex was more commonly found than an exaggerated one, contrary to the usual belief. These findings show the importance of lumbar puncture in the diagnosis of mental and nervous diseases, as neither the presence nor the absence of pupillary and tendon reflex disturbances is sufficient to determine a diagnosis.

C. W. Forsyth. 